\title{
Synthèse
}

\section{La révolution génomique au service de la filière viande bovine}

Jean-François Hocquette ${ }^{1}$

Hubert Levéziel $^{2}$

Gilles Renand ${ }^{3}$

Alain Malafosse ${ }^{4}$

${ }^{1}$ Institut national de la recherche agronomique (Inra),

UR 1213,

Unité de recherches sur les herbivores,

Theix,

63122 Saint-Genès-Champanelle

<hocquet@clermont.inra.fr>

2 Unité de génétique moléculaire animale, UMR 1061,

Institut national de la recherche agronomique (Inra),

Université de Limoges,

Faculté des sciences et techniques,

123, avenue Albert Thomas,

87060 Limoges

<hubert.leveziel@unilim.fr>

${ }^{3}$ Institut national de la recherche agronomique (Inra),

UR 337,

Station de génétique quantitative

et appliquée,

78952 Jouy-en-Josas

<ugengre@dga2.jouy.inra.fr>

${ }^{4}$ Union nationale des coopératives agricoles d'élevage et d'insémination animale (UNCEIA),

149, rue de Bercy,

75595 Paris cedex 12

<Alain.Malafosse@unceia.fr>

\begin{abstract}
Résumé
La génomique est une véritable révolution biologique avec de nombreuses applications potentielles dans toutes les sciences de la vie. En sciences animales, il sera possible de proposer de nouveaux outils aux filières bovines pour produire du lait ou de la viande répondant ainsi aux attentes des consommateurs. Dans le domaine de la sécurité alimentaire, des outils moléculaires sont déjà commercialisés pour la détection de la contamination des viandes. Dans le domaine de la traçabilité raciale ou individuelle des bovins, des marqueurs génétiques (c'est-à-dire de l'ADN) sont également disponibles. En génétique bovine, des marqueurs de l'ADN susceptibles d'être utilisés pour améliorer la tendreté ou le persillé de la viande ont été brevetés par des chercheurs australiens. Différents programmes en France, en Europe ou en Amérique ont pour objectif de vérifier la pertinence de ces marqueurs et d'en rechercher de nouveaux. Dans le domaine de la biologie musculaire, le suivi de l'expression des gènes au moyen de puces à ADN ou par séparation physique des protéines exprimées a conduit à identifier des gènes importants pour le développement du muscle ou qui peuvent être considérés comme de nouveaux marqueurs biologiques de la tendreté, du persillé ou du mode d'élevage. De grands programmes publics et/ou privés en Europe, Océanie et surtout Amérique du Nord ont pour ambition d'exploiter au mieux les avancées de la génomique au profit de l'élevage bovin.
\end{abstract}

Mots clés : bovin ; génomique ; muscle ; viande bovine

Thèmes : productions animales ; méthodes et outils

\section{Abstract \\ The genomics revolution and its benefits to the beef industry.}

Genomics has brought with it a true biological revolution and has a great number of potential applications in all areas of life sciences. In animal sciences, it will become possible to improve the production of milk or meat according to consumer demands. Concerning food safety, molecular tools have already been commercialized for the detection of contaminants in meat. Genetic markers i.e. DNA markers, have also been marketed to detect the origins of individual bovine animals and their breed. For the genetic selection of cattle, DNA markers likely to be employed for the selection of tenderness or marbling traits of beef have been patented by Australian scientists. Several ongoing projects in France, Europe and the USA aim at studying the relevance of these markers and finding new ones. In muscle biology, tracking gene expression with DNA chips or by physically separating the expressed proteins has led to the identification of genes that play an important role in muscle development. These genes could in turn serve as new biological markers for tenderness, marbling or rearing practices. Large-scale projects both publicly and privately funded, in Europe, the South Sea Islands, and especially in North America aim at exploiting to a maximum new advances in genomics in favour of beef production.

Key words: beef; cattle; genomics; muscles

Subjects: animal productions; tools and methods

Tirés à part : J.-F. Hocquette 
e progrès en agriculture est, et restera toujours, un élément important dans notre société. Il devra de plus en plus intégrer toute la diversité de la demande des consommateurs et des citoyens. Les objectifs d'accroissement de la production agricole et d'augmentation de la productivité ont fait place à des priorités désormais tournées vers la production de produits de qualité, sains et conçus dans le respect de l'environnement et du bien-être animal tout en intégrant de nouvelles préoccupations sociales. Les consommateurs expriment en effet une forte demande en matière de sécurité alimentaire, de santé et de qualité. Cette tendance, amorcée depuis plusieurs années, ne fera que s'amplifier à moyen terme (Hocquette et Gigli, 2005). Tels sont les défis majeurs auxquels nous devons mieux répondre dans l'avenir, en particulier en ce qui concerne la filière viande bovine. La révolution génomique que nous avons décrite dans un article précédent (Hocquette et al., 2007) est un atout très important qui nous permettra, au moins en partie, de résoudre ces grandes questions (Harlizius et al., 2004). Les outils de la génomique nous permettent en effet d'analyser simultanément l'ensemble des gènes impliqués dans la croissance, le stress, le métabolisme, la résistance aux maladies... rendant possible l'étude simultanée des grandes fonctions physiologiques. Des applications pratiques des progrès en génomique sont d'ores et déjà disponibles, et font l'objet de ce second article, mais nous n'en sommes encore qu'au début de l'utilisation des potentialités qu'offre cette véritable révolution biologique.

Afin de développer la génomique animale en France, le Groupement d'intérêt scientifique AGENAE (Analyse du GENome des Animaux d'Elevage) a été mis en place entre différents instituts de recherche publique (dont l'Institut national de la recherche agronomique, Inra) et les partenaires professionnels. Il a pour ambition, au sein de plusieurs espèces d'animaux d'élevage, dont le bovin, d'identifier la partie exprimée du génome, de développer la cartographie des génomes entiers et d'étudier la diversité génétique dans les populations animales ${ }^{1}$.

${ }^{1}$ www.inra.fr/presse/COMMUNIQUES/ agenae/sommaire.htm et w3.inra.fr/presse/le_programme_agenae.

\section{Identité et traçabilité}

Les applications pratiques de la génomique les plus connues à ce jour concernent tout d'abord les tests ADN visant à l'identification des individus et la certification de filiations. Ce qui est réalisable chez l'homme l'est également chez le bovin puisque la variabilité de la séquence de l'ADN entre individus induit que leur génotype est unique et qu'il représente un véritable " code-barres " propre à chaque individu. Le génotypage d'un certain nombre de marqueurs localisés sur cette séquence d'ADN constitue ainsi une empreinte génétique utilisable pour suivre l'origine d'un animal.

La filière a pris conscience de la nécessité de disposer d'un outil de traçabilité individuelle de l'animal de son élevage à la pièce de viande chez le détaillant. Cette traçabilité tout au long de la filière est aujourd'hui assurée par une série de documents administratifs (le passeport de l'animal, le registre d'abattage et les fiches de découpe et de facturation). Il a été montré qu'un panel de huit marqueurs génétiques (glossaire) dans l'ADN (de type microsatellite) suffit à contrôler avec une fiabilité de 99,99\% qu'une viande donnée est bien issue de tel ou tel animal (San Cristobal-Gaudy et al., 2000). Un tel outil pourrait ainsi être utilisé pour sécuriser le système actuel. Pour ce qui est de la vérification des filiations, procédure nécessaire pour sécuriser la fiabilité des opérations d'évaluation génétique, le génotypage d'une quinzaine de marqueurs du type microsatellite est actuellement couramment utilisé par LABOGENA, le laboratoire de certification des filiations reconnu par le ministère de l'Agriculture.

Une autre question importante chez les bovins est la traçabilité raciale pour garantir à la fois l'origine des animaux et celle des produits carnés. Après un long travail de sélection des races au fil des années, les races bovines sont aujourd'hui reconnues au travers de critères phénotypiques précis et standardisés comme la couleur de robe. La définition des patrons de coloration chez le bovin à l'instar des autres mammifères est régie par l'action d'une centaine de gènes. L'unité de Génétique moléculaire animale (UMR1061, Inra/université de Limoges) s'est investie depuis une dizaine d'années dans l'identification des allèles ou des combinaisons alléliques responsables des différents patrons de coloration chez le bovin afin d'élaborer des outils moléculaires de traçabilité raciale. Aujourd'hui, l'exploitation des allèles de trois gènes de coloration, le gène Extension (Rouzaud et al., 2000), le gène Silver (Girardot et al., 2003) et le gène Agouti, permet la discrimination entre différentes races bovines (tableau 1; Oulmouden et al., 2006). La détermination de ces allèles a fait l'objet de brevets (Oulmouden et al., 1999; Oulmouden et al., 2004). Il est à noter particulièrement que l'allèle Silver "si" est spécifique des animaux appartenant à la race Charolaise.

\section{Sécurité sanitaire des aliments et santé animale}

En sécurité sanitaire, ce sont les particularités qui différencient les génomes de diverses espèces qui sont exploitées : par exemple, une société commerciale vend une puce à $\mathrm{ADN}$ pour détecter des contaminations bactériologiques en se basant sur la détection de l'ADN bactérien dans les produits alimentaires (Murawska et al., 2004).

Par ailleurs, le système FoodExpert-ID ${ }^{\circledR 2}$ commercialisé par bioMérieux utilise également l'information génétique contenue dans l'ADN de l'échantillon alimentaire analysé. Il est basé sur une puce ADN Affymetrix à haute densité. Cette GeneChip ${ }^{\circledR}$ contient 80000 sondes oligonucléotidiques synthétisées par photolithographie sur une surface en verre de $1 \mathrm{~cm}^{2}$. Ces sondes sont complémentaires de séquences spécifiques du gène du cytochrome $b$ des vertébrés. Cette combinaison unique de sondes permet d'identifier la composition en espèces animales de l'échantillon analysé. FoodExpert-ID ${ }^{\circledR}$ permet de détecter 33 espèces de vertébrés différentes, et d'identifier simultanément la présence de produits animaux dans des échantillons alimentaires au niveau de la classe (mammifère, oiseau ou poisson) et au niveau de l'espèce (bouf, poulet, saumon...). Ainsi, les possibilités ouvertes par ce test dépassent largement celles des techniques traditionnelles d'analyse de l'alimentation qui ne

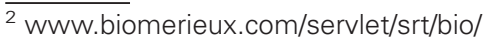
portail/dynPage?doc=PRT_PRD_IND_CTL_G_ PRD_21\&pubparams.sform $=0$ \&lang $=\mathrm{fr}$ ).
} 


\section{Tableau 1. Races et groupes de races identifiables actuellement par les tests ADN basés sur la combinatoire des variants alléliques des gènes Extension (4 alléles), Silver (3 allèles) et Agouti (2 allèles).}

Table 1. Breeds and groups of breeds currently identifiable by DNA tests based on the combination of allelic variants of the genes Extension (4 alleles), Silver (3 alleles) and Agouti (2 alleles).

\begin{tabular}{|c|c|c|c|c|c|c|c|c|c|c|c|c|}
\hline & \multirow{3}{*}{$\begin{array}{c}\text { Races } \\
\text { bovines }\end{array}$} & \multicolumn{9}{|c|}{ Gène } & \multirow{3}{*}{\multicolumn{2}{|c|}{$\begin{array}{l}\text { Combinaison } \\
\text { allélique }\end{array}$}} \\
\hline & & \multicolumn{4}{|c|}{ Extension $/ M C 1 R$} & \multicolumn{3}{|c|}{ Silver } & \multicolumn{2}{|c|}{ Agouti } & & \\
\hline & & E & $E_{1}$ & $E^{D}$ & $e$ & SI & si & $s i_{1}$ & $A$ & $A^{b r}$ & & \\
\hline \multirow{7}{*}{$\begin{array}{l}\text { \& } \\
\frac{8}{2} \\
\frac{\pi}{\pi}\end{array}$} & Limousine & & & & + & + & & & + & & I & \\
\hline & Salers & & & & + & + & & & + & & I & \\
\hline & Charolaise & & & & + & & + & & + & & II & \\
\hline & Aubrac & & + & & & + & & & + & & III & \\
\hline & Gasconne & & + & & & + & & & + & & III & \\
\hline & Parthenaise & + & & & & + & & & + & & IV & \\
\hline & $\begin{array}{l}\text { Blonde } \\
\text { d'Aquitaine }\end{array}$ & & & & + & + & & + & & & V & \\
\hline \multirow{4}{*}{ 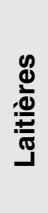 } & Simmental & & & & + & + & & + & & & V & \\
\hline & Prim'Holstein & & & + & & + & & & + & & VI & \\
\hline & Normande & + & & & & & & & & + & VII & \\
\hline & Montbéliarde & & & & + & + & & & + & + & VIII & \\
\hline
\end{tabular}

La seule race identifiable par la présence d'un allèle spécifique est la race Charolaise ; dans les autres cas, une combinaison d'allèles doit être utilisée, l'allèle $E^{D}$ étant aussi présent dans les races Angus ou Vosgienne par exemple (d'après Oulmouden et al., 2006).

détectent qu'un nombre limité d'espèces, et qui sont moins fiables, notamment lorsque des matières provenant de différentes espèces sont mélangées dans un seul produit. De plus, ce système fonctionne aussi bien sur des produits crus que sur les produits transformés ou cuits. Le rapport généré par le logiciel de FoodExpert-ID ${ }^{\circledR}$ est donc une véritable carte d'identité (Identity Card, d'où le nom "ID " du test) des espèces animales qui composent un produit, apportant ainsi une aide à la vérification de l'étiquetage de ce produit. Le système renforce donc la traçabilité et l'assurance qualité de l'industrie agroalimentaire, en permettant d'identifier les espèces animales à chaque étape des filières, " de la fourche à la fourchette".

Dans le domaine de la santé animale, l'Inra coordonne le réseau européen EADGENE 3 (European Animal Disease Genomics). Le bétail européen est vulnérable aux maladies car les agents pathogènes deviennent de plus en plus

\footnotetext{
${ }^{3}$ www.eadgene.org/.
}

résistants alors que l'usage des antibiotiques est de moins en moins souhaité. L'objectif de ce programme est donc de maintenir les animaux en bonne santé, de prévenir les maladies et de mettre au point de nouveaux vaccins ainsi que des méthodes rapides de diagnostic des infections.

\section{Amélioration}

\section{génétique de la qualité de la viande}

La sélection génétique pour améliorer les critères de qualité de la viande se heurte à la difficulté de mesurer en routine ces critères de qualité. Une alternative consiste à sélectionner sur la base de polymorphismes de gènes affectant la qualité de la viande (Renand et al. 2003). Certains tests génétiques sont déjà commercialisés par des pays étrangers tels que l'Australie pour améliorer le persillé ou la tendreté de la viande bovine (Barendse, 1997; Barendse, 2001 ; Barendse, 2003). (tableau 2). Mais ces tests ne sont valables que dans les conditions où ils ont été validés (races, types de production, mode de consommation). La France est actuellement impliquée dans différents programmes de recherche sur la qualité de la viande qui offriront, à terme, la possibilité de multiples applications.

\section{GEMQUAL}

Le programme européen GeMQual ${ }^{4}$ (Genetics of Meat Quality) récemment terminé avait pour objectif l'identification de gènes contrôlant une partie de la variabilité des caractéristiques de qualité des viandes bovines. Il reposait sur des lots de 30 taurillons non apparentés représentatifs de 15 races bovines à viande européennes, et élevés dans des conditions comparables. L'objectif du programme, était la mise en évidence et l'utilisation de

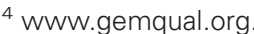




\section{Tableau 2. Tests ADN commercialisés dans le but d'améliorer la qualité de la viande bovine (d'après Hocquette et al., 2007).}

Table 2. Commercialised DNA tests for Cattle with the objective to improve beef quality (from Hocquette et al., 2007).

\begin{tabular}{|c|c|c|c|c|}
\hline Gène & Caractère & Année $^{a}$ & Découvreur & Vendeur \\
\hline $\mathrm{TG}$ & Persillé de la viande & 2000 & CSIRO/MLA, Australie & Genetic Solutions P/L \\
\hline CAST & Tendreté de la viande & 2002 & $\begin{array}{c}\text { CSIRO/MLA/Beef CRC, } \\
\text { Australie }\end{array}$ & Genetic Solutions P/L \\
\hline CAPN1 & Tendreté & 2003 & $\begin{array}{l}\text { USSDA/AgResearch NZ } \\
\text { (États-Unis, Nouvelle } \\
\text { Zélande) }\end{array}$ & Open \\
\hline $\mathrm{GH} 1$ & Persillé & 2003 & NIAS, Japon & Prescribe Genomics CO \\
\hline LEP & Persillé & 2003 & $\begin{array}{l}\text { Université de } \\
\text { Saskatchewan, Canada }\end{array}$ & Merial \\
\hline $\begin{array}{l}\text { Tests } \\
\text { multiples }\end{array}$ & Tendreté & $\begin{array}{l}2003- \\
2004\end{array}$ & & Genetic Solutions P/L \\
\hline$S C D$ & $\begin{array}{l}\text { Composition } \\
\text { en acides gras }\end{array}$ & 2004 & Université de Kobé, Japon & Prescribe Genomics CO \\
\hline $\begin{array}{l}\text { Tests } \\
\text { multiples }\end{array}$ & Persillé & 2004 & & Genetic Solutions P/L \\
\hline CAPN3 & Tendreté & 2006 & $\begin{array}{c}\text { CSIRO/MLA/Beef CRC, } \\
\text { Australie }\end{array}$ & Genetic Solutions P/L \\
\hline
\end{tabular}

polymorphismes de type "mutations ponctuelles " (SNP) à l'intérieur ou proches de gènes d'intérêt choisis sur la base de leur fonction physiologique ; ce programme correspondait plutôt à un travail de criblage raisonné pour identifier les gènes les plus intéressants parmi un grand nombre. Au total, près de 400 gènes auront été examinés et environ 710 polymorphismes ont été mis en évidence dans 209 d'entre eux. Le génotypage des animaux pour les SNP considérés a été réalisé et l'analyse statistique en cours permettra de détecter l'existence d'éventuelles relations entre certains allèles et les caractéristiques qualitatives des viandes (Levéziel et al., 2005).

\section{QUALVIGENE}

Le programme français QUALVIGENE porté par l'Union nationale des coopératives agricoles d'élevage et d'insémination animale (UNCEIA) et financé par l'AGENAE s'appuie sur plus de 3000 taurillons engraissés puis abattus dans des conditions contrôlées en races Charolaise, Limousine et Blonde d'Aquitaine. Ce programme mobilise 24 partenaires de la profession, de l'Institut de l'élevage ou de l'Inra. Un de ses objectifs est de tester, avec nos principales races bovines à viande françaises, la pertinence des tests ADN proposés par d'autres partenaires, notamment les chercheurs australiens, ou des gènes mis en évidence dans d'autres programmes comme GEMQUAL. QUALVIGENE constitue non seulement un programme de validation, mais représente aussi un investissement sur le long terme afin d'exploiter dans le futur d'autres retombées scientifiques grâce à l'existence de la banque de données constituée (phénotypes enregistrés) et de l'ADN toujours disponible (Dodelin, 2006). En particulier, ce programme QUALVIGENE permettra aussi la détection de nouveaux $Q T L$ associés à la qualité de la viande bovine.

\section{MUGENE}

Le projet MUGENE porté par l'Inra et financé par AGENAE va apporter d'autres informations sur la biologie du muscle et la qualité de la viande. Ce programme concerne deux lignées de bovins Charolais de l'Inra sélectionnées de façon divergente selon leur potentiel de croissance musculaire. Les animaux sont des taurillons alimentés à l'auge ou des bœufs conduits au pâturage de façon à analyser conjointement l'effet de la sélection génétique, du mode de production et l'interaction entre la génétique et les facteurs d'élevage. Ce projet, qui repose sur un dispositif expérimental de l'Inra, constitue tout d'abord une approche de criblage de l'ensemble du génome par la mise en ouvre de marqueurs génétiques couvrant l'ensemble des chromosomes bovins. Il permettra aussi une ana- lyse fine des caractéristiques musculaires, observées sur deux muscles pour lesquels les mêmes mesures que dans QUALVIGENE seront réalisées. Les approches de génomique fonctionnelle (transcriptome et protéome) seront également mises en œuvre. Ce projet devrait aboutir notamment à la mise en évidence de marqueurs génétiques et biologiques du potentiel de croissance des animaux ainsi que de la tendreté et de la flaveur de leur viande ${ }^{5}$.

\section{Perspectives}

La finalité de ces recherches est de proposer des outils moléculaires de sélection et de diagnostic des caractéristiques du muscle et des qualités de la viande (tendreté, goût). Nous pouvons ainsi imaginer pouvoir orienter à terme la sélection les bovins vers le type de production le plus adapté à la demande des consommateurs, ou orienter les carcasses, après un diagnostic lors de l'abattage, vers des traitements visant à optimiser le temps et les conditions de maturation de la viande afin de valoriser au mieux le potentiel de leur muscle à produire une viande de qualité.

\footnotetext{
${ }^{5}$ www.clermont.inra.fr/commissionbovine/textes/2003picard.pdf.
} 


\section{Génomique fonctionnelle du muscle de bovin}

Les premiers travaux réalisés jusqu'à présent sur le transcriptome (ensemble des transcrits) et le protéome (ensemble des protéines) du muscle de bovin ont porté sur la construction de la qualité de la viande tout au long de la vie de l'animal, de sa conception à l'abattage. Ils ont été principalement réalisés aux États-Unis, en France, et en Australie et ont fait l'objet d'un article de synthèse récent (Hocquette et al. 2007).

\section{Étude du transcriptome}

Aux États-Unis, une étude a été réalisée pour comparer l'expression des gènes de jeunes embryons bovins de type normal ou de type culard, les bovins culards étant caractérisés par une hypertrophie musculaire due à une mutation du gène de la myostatine qui est un facteur de croissance régulant négativement la masse musculaire. Les gènes différentiellement exprimés entre les deux types génétiques concernent des facteurs de transcription, des gènes impliqués dans la synthèse ou la dégradation des protéines, dans la prolifération cellulaire ou dans le métabolisme. Certains de ces gènes ont été localisés sur le chromosome 5 et sont physiquement proches d'un QTL associé à la dureté de la viande (Potts et al., 2003). Il s'agit d'une première étape dans la compréhension des mécanismes biologiques associant l'hypertrophie musculaire à une meilleure tendreté de la viande.

En France, le transcriptome a été étudié dans le cadre de quatre expérimentations conduites par l'Inra de Theix (Unité de recherches sur les herbivores) portant sur : i) la mise en place des caractéristiques du muscle au cours de son développement foetal et postnatal ; ii) les effets sur les caractéristiques musculaires d'une sélection génétique sur le potentiel de croissance musculaire ; iii) la comparaison entre une conduite au pâturage et une alimentation à l'auge à base d'ensilage de maïs ; et iv) la comparaison d'échantillons de muscles produisant une viande tendre ou dure. Pour les trois premiers modèles, nous avons analysé le transcriptome d'un muscle plutôt lent oxydatif, le rectus abdominis (RA), correspondant à la bavette de flanchet et d'un muscle rapide glycolytique, le semitendinosus (ST), correspondant au rond de gîte.

En ce qui concerne le développement du muscle, nous avons détecté environ 110 gènes différentiellement exprimés entre 110, 180, 210 et 260 jours de vie fotale et 15 mois d'âge postnatal. C'est au cours du dernier tiers de vie foetale et jusqu'à l'âge de 15 mois que nous avons détecté le plus de gènes différentiellement exprimés (Sudre et al., 2003). Ce résultat est en accord avec nos connaissances actuelles de la myogenèse chez le bovin car cette période correspond à une différenciation importante des fibres musculaires (Picard et al., 2002).

En ce qui concerne le modèle de croissance musculaire, des gènes différentiellement exprimés ont été détectés dans l'un ou l'autre des muscles RA et ST lors des analyses comparant les deux lignées de taurillons Charolais sélectionnées de façon divergente sur la base de leur potentiel de croissance musculaire (Sudre et al., 2005). Ces gènes concernent essentiellement des protéines impliquées dans la structure du muscle ou la régulation cellulaire. Des expériences complémentaires ont montré que certains gènes correspondant aux caractéristiques rapides glycolytiques des fibres musculaires sont davantage exprimés chez les bovins à fort potentiel de croissance musculaire, facteur favorable pour la maturation de la viande et donc pour sa tendreté (CassarMalek et al., 2005a).

En ce qui concerne la comparaison des modes d'élevage, nous avons mis en évidence des gènes différentiellement exprimés en fonction du type de conduite des animaux (ensilage de maïs à l'auge versus herbe pâturée). Les différences d'expression d'au moins l'un de ces gènes pourraient correspondre à des apports alimentaires différents en sélénium ou à une activité physique accrue des animaux au pâturage (Cassar-Malek et al., 2005b).

De plus, quel que soit le modèle animal, nous avons détecté des gènes différentiellement exprimés entre les muscles RA et ST qui permettent de mieux comprendre le déterminisme des différences physiologiques entre types de muscles (Hocquette et al., 2007). Ces marqueurs biologiques du type de muscle sont importants dans la mesure où les différences de qualité de la viande bovine (tendreté notamment) diffèrent plus entre muscles au sein d'un même animal qu'entre animaux.
En ce qui concerne la qualité de la viande, nous avons mis en évidence, dans le cadre du projet MUGENE, un gène (DNAJA1) dont le niveau d'expression dans le muscle long dorsal à l'abattage est négativement corrélé à la note de tendreté de la viande appréciée par un jury d'analyse sensorielle après 14 jours de maturation. À partir d'échantillons du muscle long dorsal prélevés sur 14 taurillons de race Charolaise, le niveau d'expression de DNAJA1 explique, à lui seul, près de $65 \%$ de la variabilité de la tendreté, d'où le dépôt d'un brevet (Bernard et al., 2006 ; Bernard et al., 2007). DNAJA1 code pour une protéine qui est membre de la famille des heat shock protéines de $40 \mathrm{kDa}$ et qui pourrait jouer un rôle dans l'importation des protéines dans la mitochondrie. L'importance de ce gène dans le déterminisme de la tendreté doit être confirmée sur un plus grand nombre d'animaux, pour d'autres types de muscles, pour d'autres races bovines et pour différents modes d'élevage.

Parmi les gènes différentiellement exprimés au cours du développement ou entre muscles, certains ont une fonction biologique connue, mais pourtant leur rôle dans la biologie du muscle de bovin n'avait jamais été observé (tel est le cas de DNAJA1). Par ailleurs, environ un tiers des gènes différentiellement exprimés n'ont pas encore de fonctions biologiques connues. Bien qu'il s'agisse des premiers pas de la génomique fonctionnelle bovine, ces constats démontrent que les études actuelles ouvrent de nouvelles pistes de recherche, et devraient conduire vers l'identification de gènes indicateurs des caractéristiques musculaires (Bernard et al., 2007).

En Australie, des gènes différentiellement exprimés ont été mis en évidence entre des bovins de race Holstein et des bovins de race Noire Japonaise caractérisés par leur aptitude à produire une viande persillée, c'est-à-dire riche en lipides intramusculaires. Les gènes préférentiellement exprimés chez les Noirs Japonais sont impliqués dans la mise en place du tissu adipeux ou la synthèse des lipides tandis que les gènes exprimés chez les bovins Holstein sont plutôt impliqués dans le développement du muscle ou les caractéristiques du tissu conjonctif (Wang et al., 2005).

\section{Étude du protéome}

L'analyse du protéome consiste à extraire les protéines musculaires et à les séparer par électrophorèse bidimensionnelle. 
Une carte protéique du muscle ST bovin a été établie (Bouley et al., 2004a). Cette carte, qui sera complétée progressivement, servira de support pour l'étude du protéome du muscle de bovin dans le cadre de différentes problématiques (développement musculaire, qualité de la viande). L'analyse des résultats indique que les variations quantitatives et qualitatives des protéines du muscle ne concernent qu'une très faible proportion d'entre elles (inférieure à $10 \%$ ).

Une première étude a mis en évidence une sous-expression des isoformes lentes de la troponine $\mathrm{T}$ squelettique dans le muscle de bovins présentant une hypertrophie musculaire (Bouley et al., 2005). L'expression différentielle des isoformes de troponine $\mathrm{T}$ et de la protéine de liaison des acides gras est cohérente avec une proportion plus élevée de fibres rapides glycolytiques dans les muscles des bovins culards aux muscles hypertrophiés, facteur favorisant la maturation de la viande, et donc sa tendreté.

Une seconde étude a porté sur l'analyse protéomique de muscles de taurillons de 15 mois de trois races différentes, dont le rond de gîte (ST), muscles qui avaient été préalablement classés dans deux catégories de tendreté de leur viande : forte ou faible. Cette étude a permis d'identifier de nouveaux marqueurs biologiques de la tendreté (parvalbumine, chaînes légères de myosine, protéine de liaison des acyl-CoA...) qui diffèrent toutefois entre races (Bouley et al., 2004b).

\section{Étude de la structure des gènes}

Le développement de ces analyses du transcritptome ou du protéome représente en fait une démarche de criblage afin de repérer les gènes influençant la mise en place des phénotypes d'intérêt pour les producteurs ou les consommateurs. L'étape suivante consiste à étudier ces gènes plus en détail pour identifier des polymorphismes éventuels, déterminer la régulation de leur expression ou mieux connaitre leur fonction, afin d'élaborer des outils utilisables, notamment des marqueurs $A D N$ exploitables pour la sélection génétique ou des marqueurs biologiques pouvant aider à la gestion des systèmes d'élevage. Pour certains gènes déjà identifiés, les travaux conduits en France montrent que la situation est certainement plus complexe qu'imaginé. Ainsi, par exemple, les travaux de l'Inra et de l'université de
Limoges ont porté sur le gène de la calpastatine, qui joue un rôle essentiel dans le déterminisme de la tendreté de la viande bovine : en effet, la calpastatine inhibe l'activité des calpaïnes qui sont les principales protéases intervenant dans la protéolyse ménagée des protéines musculaires au cours de la maturation du muscle et de sa transformation en viande. Ce gène est morcelé en 35 parties codantes (ou exons) réparties sur une zone de 140000 bases de l'ADN. Son expression est sous la dépendance de plusieurs promoteurs qui contrôlent la synthèse de plusieurs familles de transcrits, qui peuvent eux-mêmes subir diverses modifications. Plusieurs protéines (dont le nombre n'est pas encore déterminé avec certitude) aux propriétés éventuellement différentes (concernant par exemple l'inhibition des calpaïnes) peuvent être ainsi générées dans les cellules (Raynaud et al., 2005a; Raynaud et al., 2005b). D'autres travaux en cours pour d'autres gènes montrent également des situations complexes avec souvent de véritables originalités pour l'espèce bovine.

\section{Conclusion}

D’une façon générale, les méthodes de la génomique peuvent être soit appliquées selon une approche systématique pour étudier l'ensemble des gènes, des transcrits ou des protéines, soit, au contraire, déployées de manière plus ciblée pour étudier une région du génome ou quelques gènes particuliers. Dans tous les cas, ces méthodes nécessitent encore de nombreux efforts méthodologiques mais confèrent néanmoins aux équipes de recherche une capacité d'investigation jusque-là inégalée et porteuse de réelles espérances pour l'élevage en général.

En ce qui concerne la viande, l'objectif à long terme en génétique animale est de disposer d'un ensemble de marqueurs génétiques pour améliorer la sélection d'animaux ayant le potentiel de produire une viande de qualité élevée et constante. Les premiers marqueurs génétiques sont actuellement commercialisés par des collègues australiens mais leur pertinence pour nos races bovines européennes dans nos propres conditions d'élevage est en cours d'étude.

Pour les producteurs de viande, l'objectif est d'exploiter la variabilité des caractères intrarace et interraces pour produire une viande de bonne qualité. En d'autres termes, les expérimentations en cours permettront de retenir des "prédicteurs biologiques" des caractéristiques des muscles et des viandes qui différent entre pièces de boucherie, entre animaux et entre races.

Les premiers travaux de génomique fonctionnelle ont confirmé que les bovins à fort potentiel de croissance musculaire ont des muscles plus rapides et plus glycolytiques, ce qui peut potentiellement favoriser la maturation du muscle et ainsi la tendreté de la viande produite. Des marqueurs biologiques du type de muscle et de l'hypertrophie musculaire ou encore des gènes spécifiquement régulés en fonction du système d'élevage et au cours de la croissance du muscle ont été mis en évidence. De nouveaux indicateurs de la tendreté de la viande ont également été révélés par l'approche protéomique ou transcriptomique et d'autres sont en cours d'étude plus approfondie.

\section{Petit glossaire pour décrypter ce dossier génomique \\ (en complément du glossaire publié dans le précédent article Hocquette et al., 2007)}

QTL (Quantitative Trait Locus) : région de I'ADN (locus) associée à un caractère phénotypique particulier. La variation allélique d'un marqueur du locus est associée à la variabilité du caractère considéré.

Marqueur génétique (ou marqueur ADN): segment d'ADN polymorphe, facilement identifiable par génotypage et dont la position sur un chromosome est connue. Ce marqueur peut ainsi permettre de localiser un gène voisin ou être directement dans une séquence codante d'un gène. Un marqueur génétique est donc une " étiquette " sur la chaîne d'ADN, facile à observer, et qui renseigne sur le génotype de l'individu qui le porte et sur son potentiel pour réaliser un phénotype.

Marqueur biologique: caractéristique de l'expression d'un gène, observée au niveau des transcrits ou des protéines et associée à une particularité du phénotype. 


\section{Références}

Dodelin V. Qualvigène: un programme de recherche de gènes impliqués dans les qualités de la viande. Bovins Limousins 2006 ; 166 17-23.

Barendse W. Assessing lipid metabolism Patent Publication WO9923248, Patent US 6383751 . Available : http ://ep.espacenet.com/. 1997. International Publication date : 14 May 1999.

Barendse W. DNA markers for meat tender ness. Patent WO02064820. Patent Application PCT/AU02/00122. http ://ep.espacenet.com/ 2001. Accessed : 22 August 2002.

Barendse W. DNA markers for marbling. Patent Publication WO2004070055. Available http ://ep.espacenet.com/. 2003. Accessed : 19 August 2004.

Bernard C, Cassar-Malek I, Hocquette JF.Genomic marker for meat tenderness. Brevet 06 300943.5 Déposé le 12 septembre 2006.

Bernard C, Cassar-Malek I, Le Cunff M, Dubrœucq H, Renand G, Hocquette JF. New indicators of beef sensory quality revealed by expression of specific genes. J Agric Food Chem 2007 ; $55: 5229-37$.

Bouley J, Chambon C, Picard B. Mapping of bovine skeletal muscle proteins using two dimensional gel electrophoresis and mass spectrometry. Proteomics $2004 ; 4$ : 1811-24.

Bouley J, Meunier B, Chambon C, De Smet S, Hocquette JF, Picard B. Proteomic analysis of bovine skeletal muscle hypertrophy. Proteo mics $2005 ; 5: 490-500$.

Bouley J, Meunier B, Culioli J, Picard B. Analyse protéomique du muscle de Bovin appliquée à la recherche de marqueurs de la tendreté de la viande. Renc Rech Ruminants $2004 ; 11: 87-9$.

Cassar-Malek I, Ueda Y, Bernard C, et al. Molecular and biochemical muscle characteristics of Charolais bulls divergently selected for muscle growth. In : Hocquette JF, Gigli S, eds. Indicators of milk and beef quality EAAP Publication, 112. Wageningen (Pays Bas): Wageningen Academic Publishers, 2005.
Cassar-Malek I, Bernard C, Jurie C, et al. Pasture-based beef production systems may influence muscle characteristics and gene expression. In : Hocquette JF, Gigli S, eds. Indicators of milk and beef quality. EAAP Publication, 112. Wageningen (Pays Bas) Wageningen Academic Publishers, 2005.

Girardot M, Guibert S, Laforet MP, Levéziel H, Julien R, Oulmouden A. Exploitation des gènes de la coloration de la robe pour une traçabilité raciale des produits d'origine bovine. Renc Rech Ruminants 2003 ; 10 : 33-6. Harlizius B, van Wijk R, Merks JWM. Geno-
mics for food safety and sustainable animal production. J Biotechnol 2004 ; 113 : 33-42.

Hocquette JF, Gigli S. The challenge of quality. In: Hocquette JF Gigli S, eds Indicators of milk and beef quality. EAAP Publication, 112. milk and beef quality. EAAP Publication, 112.
Wageningen (Pays Bas): Wageningen AcadeWageningen (Pays Bas)
mic Publishers, 2005.

Hocquette JF, Lehnert S, Barendse W, Cassar Malek I, Picard B. Recent advances in cattle functional genomics and their application to beef quality. Animal $2007 ; 1$ : 159-73.

Hocquette JF, Levéziel H, Renand G, Malafosse $A$. La révolution génomique concerne aussi le bovin. Cah Agric 2007 ; 16: 163-9.

Levéziel $\mathrm{H}$, Amarger $\mathrm{V}$, Delourme $\mathrm{D}$, et al. Gènes bovins et qualités des viandes : présentation du programme européen GEMQUAL. Neuvièmes journées des sciences du muscle et technologies de la viande, ClermontFerrand (France), 15-16 octobre 2002. Viandes Prod Carnés 2005 ; HS : 151-2.

Murawska A, Tyburski J, Budzyński T, Jarkiewicz-Tretyn J, Donderski W, Tretyn A Applying the microarray (chip) DNA technique for bacteriological examinations of foodstuffs. Med Weter 2004 ; 60: 481-4.

Oulmouden A, Levéziel H, Julien R. À quo peut bien servir de connaître les gènes qui contrôlent la couleur des bovins. Bovins Limousins 2006 ; $167: 20-4$.

Oulmouden A, Gallet PF, Rouzaud F, et al. Demande de brevet 98-05809 déposée auprès de I'INPI le 7 Mai 1998, aux noms conjoints de I'INRA et de I'Université de Limoges. Titre "Séquences nucléotidiques pour la mise en œuvre d'un procédé d'identification génétique de populations bovines et de produits dérivés ". Demande renouvelée le 7 Mai 1999 (99 05861) après apport de données complémentaires.
Oulmouden A, Julien R, Laforet MP, Levéziel H. Demande de brevet 03-09161 déposée auprès de I'INPI le 25 juillet 2003, aux noms conjoints de I'INRA et de I'Université de Limoges. Titre: "Utilisation du gène SILVER pour l'authentification de l'origine raciale des populations animales et de leurs produits dérivés ". Demande d'extension à l'étranger en juillet 2004 (PCT No FR04/01952 du 22/07/04).

Picard B, Lefaucheur L, Berri C, Duclos MJ. Muscle fibre ontogenesis in farm animal species. Reprod Nut Dev $2002 ; 42$ : 415-31.

Potts JK, Echternkamp SE, Smith TPL, Reecy JM. Characterization of gene expression in double-muscled and normal-muscled bovine embryos. Anim Genet 2003 ; 34 : 438-44.

Raynaud P, Gillard M, Parr T, Bardsley R, Amarger V, Levéziel $\mathrm{H}$. Correlation between calpastatin mRNA transcripts and protein isoforms. Arch Biochem Biophys 2005; 440: 46-53.

Raynaud $\mathrm{P}$, Jayat-Vignoles $\mathrm{C}$, Laforêt MP, Levéziel $\mathrm{H}$, Amarger $\mathrm{V}$. Four promoters direct expression of the bovine calpastatin gene. Arch Biochem Biophys 2005 ; 437 : 69-77.

Renand G, Larzul C, Le Bihan-Duval E, Le Roy $P$. L'amélioration génétique de la qualité de la viande dans les différentes espèces: situation actuelle et perspectives à court et moyen terme. INRA Prod Anim 2003; 16 : 159 73.

Rouzaud F, Martin J, Gallet PF, et al. A first genotyping assay of French cattle breeds based on a new allele of the extension gen encoding the melenocortin-1 receptor (Mc1r). Genet Sel Evol 2000 ; 32 : 511-20.

San Cristobal-Gaudy M, Renand G, Amigues $Y$, Bosher MY, Levéziel $H$, Bibé $B$. Traçbilité individuelle des viandes bovines à l'aide de marqueurs génétiques. INRA Prod Anim $2000 ; 13: 269-76$.

Sudre K, Cassar-Malek I, Listrat A, et al. Biochemical and transcriptomic analyses of two bovine skeletal muscles in Charolais bulls divergently selected for muscle growth. Meat Sci $2005 ; 70: 267-77$.

Sudre K, Leroux C, Piétu G, et al. Transcriptome analysis of two bovine muscles during ontogenesis. J Biochem 2003 ; 133 : 745-56.

Wang YH, Reverter A, Mannen H, et al. Transcriptional profiling of adult muscle in Japanese Black Cattle to identify genes involved with the development of intramuscular fat. Austr J Exp Agr 2005 ; $16: 201-10$. 